\title{
Biochemical identification techniques and antibiotic susceptibility profile of lipolytic ambiental bacteria from effluents
}

\author{
A. F. G. Rave ${ }^{a}$, A. V. Kuss ${ }^{b}$, G. H. S. Peil ${ }^{a}$, S. R. Ladeira , J. P. V. Villarreal \\ and P. S. Nascente $e^{e *}$ \\ aPrograma de Pós-graduação em Bioquímica e Bioprospecção, Laboratório de Microbiologia Ambiental, \\ Departamento de Microbiologia e Parasitologia, Instituto de Biologia, Universidade Federal de Pelotas - UFPel, \\ Av. Eliseu Maciel, IB Prédio 18, Sala 13, CEP 96160-000, Capão do Leão, RS, Brasil \\ ${ }^{b}$ Laboratório de Microbiologia Ambiental, Departamento de Microbiologia e Parasitologia, \\ Instituto de Biologia, Universidade Federal de Pelotas - UFPel, Av. Eliseu Maciel, IB Prédio 18, Sala 13, \\ CEP 96160-000, Capão do Leão, RS, Brasil \\ 'Laboratório Regional de Diagnóstico, Faculdade de Veterinária, Laboratório de Doenças Infecciosas, Universidade \\ Federal de Pelotas - UFPel, Av. Eliseu Maciel, IB Prédio 18, Sala 03, CEP 96160-000, Capão do Leão, RS, Brasil \\ ${ }^{\mathrm{d} F a c u l d a d}$ de Veterinaria y Zootecnia, Universidade Autonoma de Nuevo Leon, General Francisco Villa, s/n, \\ Ex Hacienda El Canadá, 66050, Cod. Gral Escobedo, Monterrey, NL, México \\ 'Laboratório de Micologia e Bioprospecção, Departamento de Microbiologia e Parasitologia, Instituto de Biologia, \\ Universidade Federal de Pelotas - UFPel, Av. Eliseu Maciel, IB Prédio 18, Sala 03, CEP 96160-000, \\ Capão do Leão, RS, Brasil \\ *e-mail: pattsn@gmail.com
}

Received: October 19, 2017 - Accepted: April 09, 2018 - Distributed: November 30, 2019

\begin{abstract}
Different methodologies have been developed throughout the years to identify environmental microorganisms to improve bioremediation techniques, determine susceptibility profiles of bacteria in contaminated environments, and reduce the impact of microorganisms in ecosystems. Two methods of bacterial biochemical identification are compared and the susceptibility profile of bacteria, isolated from residential and industrial wastewater, is determined. Twenty-four bacteria were retrieved from the bacteria bank of the Environmental Microbiology Laboratory at the Institute of Biology (IB) of the Universidade Federal de Pelotas, Pelotas, Brazil. Bacteria were identified by conventional biochemical tests and by the VITEK ${ }^{\circledR} 2$ automated system. Further, the susceptibility profile to antibiotics was also determined by the automated system. Six species of bacteria (Raoutella planticola, K. pneumoniae ssp. pneumoniae, Serratia marcescens, Raoutella sp., E. cloacae and Klebsiella oxytoca) were identified by conventional biochemical tests, while three species of bacteria (K. pneumoniae ssp. pneumoniae, S. marcescens and K. oxytoca) were identified by VITEK ${ }^{\circledR}$ automated system. VITEK ${ }^{\circledR} 2$ indicated agreement in $19(79.17 \%)$ isolates and difference in five $(20.83 \%)$ isolates when compared to results from conventional biochemical tests. Further, antibiotic susceptibility profile results showed that all isolates $(100 \%)$ were resistant to at least one out of the 18 antibiotics tested by VITEK ${ }^{\circledR} 2$. Thus, no multi-resistant bacteria that may be used in effluent treatment systems or in bioremediation processes have been reported. Results indicate VITEK ${ }^{\circledR} 2$ automated system as a potential methodology in the determination of susceptibility profile and identification of environmental bacteria.
\end{abstract}

Keywords: environmental bactéria, VITEK ${ }^{\circledR} 2$, S. marcescens, microorganisms, biochemistry, multi-resistant bacteria.

\section{Técnicas de identificação bioquímica e perfil de suscetibilidade antibiótica de bactérias ambientais lipolíticas de efluentes}

\section{Resumo}

Diferentes metodologias foram desenvolvidas ao longo dos anos para identificar microrganismos ambientais para melhorar as técnicas de biorremediação, determinar perfis de suscetibilidade de bactérias em ambientes contaminados e reduzir o impacto de microrganismos nos ecossistemas. Dois métodos de identificação bioquímica bacteriana são comparados e o perfil de susceptibilidade de bactérias, isoladas de efluentes residenciais e industriais, é determinado. Vinte e quatro bactérias foram coletadas do banco de bactérias do Laboratório de Microbiologia Ambiental do Instituto de Biologia (IB) da Universidade Federal de Pelotas, Pelotas, Brasil. As bactérias foram identificadas por testes bioquímicos convencionais e pelo sistema automatizado VITEK ${ }^{\circledR} 2$. Além disso, o perfil de suscetibilidade aos antibióticos também foi determinado pelo sistema automatizado. Seis espécies de bactérias (Raoutella planticola, K. pneumoniae ssp. pneumoniae, 
Serratia marcescens, Raoutella sp., E. cloacae e Klebsiella oxytoca) foram identificadas por testes bioquímicos convencionais, enquanto três espécies de bactérias (K. pneumoniae ssp. pneumoniae, S. marcescens e K. oxytoca) foram identificados pelo sistema automatizado VITEK ${ }^{\circledR} 2$. VITEK ${ }^{\circledR} 2$ indicou concordância em $19(79,17 \%)$ isolados e diferença em cinco $(20,83 \%)$ isolados quando comparados aos resultados de testes bioquímicos convencionais. Além disso, os resultados do perfil de suscetibilidade aos antibióticos mostraram que todos os isolados (100\%) foram resistentes a pelo menos um dos 18 antibióticos testados pelo VITEK ${ }^{\circledR}$. Assim, não foram relatadas bactérias multirresistentes que possam ser usadas em sistemas de tratamento de efluentes ou em processos de biorremediação. Os resultados indicam que o sistema automatizado VITEK ${ }^{\circledR} 2$ é uma metodologia potencial na determinação do perfil de suscetibilidade e identificação de bactérias ambientais.

Palavras-chave: bactérias ambientais, VITEK ${ }^{\circledR} 2$, S. marcescens, microorganismos, bioquímica, bactérias multirresistentes.

\section{Introduction}

Several anthropic activities are increasing residual levels worldwide. There is great concern on soil and water due to increasing pollution as a result of different industrial, mineralization, agriculture and domestic processes everywhere (Ren et al., 2009). Main environmental impacts by organic solid wastes originate from fermentation of waste. The fermentation produces organic acid formation, contributing towards unpleasant odors and decrease of oxygen in the water, with damages to water animals (Graminha et al., 2008).

Further, other types of wastes, such as oil and fat effluents from residences, restaurants and food industries, are on the increase due to the human feeding processes. The lipids, indissoluble in water and highly stable, in the effluents are the result of industrial activities and are compounds that greatly impact the environment (Hasan et al., 2006; Odeyemi et al., 2013). These contaminants remain on the water surface in the form of foams and bubbles (Olivo et al., 2010; Polonio et al.,2014).

The microorganisms' metabolic process is responsible for the removal of contaminants (Mandri and Lin, 2007) by reducing their concentration (Bello, 2007) or by transforming pollutants into compounds with low toxicity (Colla and Costa, 2003; Mongkolthanaruk and Dharmsthiti, 2002).

During decades, bacteria have been routinely identified in laboratories by biochemical tests and by microscopic identification, which, as a rule, need time for their execution, ranging from a couple of hours to several days (Martiny et al., 2012). Since bacteria do not have enough morphological characteristics to pinpoint their peculiarities, several methodologies have been developed to test their metabolic or enzymatic reactions, enabling grouping and identification at genus or species level (Busch and Nitschko, 1999; Romeu et al., 2010).

Conventional analyses are time-consuming techniques and semi-automatic methods require great amounts of biologic material. In fact, the above constitute significant disadvantages for the identification of microorganisms (Martiny et al., 2012).

The VITEK (bioMérieux) System is a useful tool to identify different groups of bacteria by a miniaturized biochemical test with specific cards containing 64 fluorescent biochemical tests. Although the existence of few research works reporting the use of this equipment to identify bacteria from the environment, good results have been obtained in the microorganism's identification in different ecosystems (Song and Leff, 2005; Mendes et al., 2018).

These bacteria should be studied and evaluated for their use as bioremediators, as there is an increasing need for environmental protection by contaminants that are harmful to health. However an important consideration is the knowledge about the susceptibility profile to antibiotics of these environmental bacteria so that they can be disseminated in the environment.

Antibiotic-resistant bacteria already widespread in the environment as a result of the indiscriminate use of antibiotics can be detected in different environments, such as hospital sewage, domestic sewage and contaminated river water, transforming effluents into very aggressive factors for the environment (Abreu et al., 2010).

Due to its importance in the identification of environmental bacteria and determination of their susceptibility profile, the current research aimed to compare two techniques of identification: conventional and automated and to evaluate the susceptibility to antibiotics of environmental bacteria in the region of Pelotas RS Brazil.

\section{Materials and Methods}

\subsection{Sample origin and processing}

A study by Peil et al. (2016) verified the lipolytic characteristics of the isolated bacteria used in current research. These bacteria were stored in the Laboratory of Environmental Microbiology of the Biology Institute of UFPel.

Twenty-four Gram-negative bacteria were selected: five residues (isolates 1, 2, 3, 4 and 5), five commercial isolates (isolates $6,7,8,9$ and 10) and 14 of industrial effluent isolates).

The final identification obtained by each technique was compared individually considering that the equivalence above $90 \%$ is satisfactory (Bamford et al., 2010).

\subsection{Identification and morphologic and biochemical characterization}

In order to confirm the cellular morphology and classification of the bacteria, the Gram staining technique, with 100X optical microscopy visualization, should be used to determine the shape, arrangement and classification of Gram-negative bacteria. Biochemical tests and identification 
cards selected to compare as two identification techniques: conventional and automated $\left(\mathrm{VITEK}^{\circledR} 2\right)$.

\subsection{Conventional biochemical tests}

After phenotypic colony and cell identification through Gram staining by microscopic visualization were performed, the conventional biochemical tests were made to determine the isolated bacteria classification according to the Gram-negative bacterial identification protocol (Barrow and Feltham, 1993).

The conventional biochemical tests were: $\mathrm{GS}=\mathrm{Gram}$ staining; $\mathrm{OX}=$ Oxidase $\mathrm{Mc}=$ MacConkey medium; $\mathrm{CI}=$ Citrate; $\mathrm{SIM}=\mathrm{SIM}$ medium; $\mathrm{AR}=$ Arabinose $; \mathrm{CE}=$ Cellobiose; $\mathrm{RA}=$ Raffinose; $\mathrm{XY}=$ Xylose; VP = Voges-Proskauer; $\mathrm{LAC}=$ Lactose $; \mathrm{OR}=$ Ornithine; $\mathrm{DU}=$ Dulcitol.

\subsection{VITEK ${ }^{\circledR} 2$ System}

Biochemical analyses were performed by VITEK $^{\circledR} 2$ automated system. The isolated bacteria were inoculated in BHI solid culture and incubated for 24 hours at $35^{\circ} \mathrm{C}$. A homogeneous suspension with an optic density of microorganisms between 0.55 and 0.63 , using a DensiCHEK $^{\mathrm{TM}}$ VITEK $^{\circledR} 2$ Caliper, was performed for the preparation of the samples. Samples were then analyzed by VITEK $^{\circledR} 2$. Forty-eight biochemical tests were performed with Gram-negative bacterium identification cards.

The VITEK ${ }^{\circledR} 2$ biochemical tests are described in Table 1.

\subsection{Antibiotic susceptibility profile}

The antibiotic susceptibility profile was also determined by VITEK $^{\circledR} 2$ automated system. An aliquot of $145 \mu \mathrm{L}$ of cellular suspension, with an optic density between 0.55 and 0.63 , was added in $3 \mathrm{~mL}$ saline solution at $0.85 \%$. Samples were analyzed by VITEK $^{\circledR} 2$ which indicated resistance or sensibility shown by the bacteria through several antibiotic concentrations in the anti-biogram cards. Susceptibility profiles were performed with 18 antibiotics in the specific anti-biogram AST cards, specific for Gram-negative bacteria.

Os antibióticos testados pelo Sistema VITEK ${ }^{\circledR}$ nas bactérias gram-negativas foram: Ampicilina, Ampicilina/Sulbactam, Ppiperacilina/Tazobactam, Cefuroxima, Cefuroxima axetil, Cefoxitina, Ceftazidima, Ceftriaxona, Cefepima, Ertapnem, Imipenem, Meropenem, Amicacina, Gentamicina, Ciprofloxacina, Tigeciclina e Colistina.

\section{Results}

\subsection{Biochemical Conventional Identification}

Biochemical tests were performed on the 24 bacterial isolates, identified as Gram-negative, bacillus and cocci, to identify the genus and the species of the Gram-negative bacteria, following Barrow and Feltham (1993).

In the case of the 24 analyzed bacteria, the biochemical conventional tests revealed six species of bacteria (Table 2), namely, Raoutella planticola (1 isolate $-4.17 \%)$, K. pneumoniae ssp. pneumoniae (1 isolate $-4.17 \%$ ), Serratia marcescens (16 isolates $-66.67 \%)$, Enterobacter cloacae (1 isolate $-4.17 \%$ ),
Raoutella sp. (2 isolates $-8.33 \%)$ and Klebsiella oxytoca (3 isolates $-12.5 \%$ ).

\subsection{Biochemical identification by VITEK ${ }^{\circledR}$ Automated System}

Gram-negative bacterial identification cards were used, specific for Gram-negative bacteria. Three out of the 24 isolated bacteria species were identified by VITEK $^{\circledR} 2$, namely $K$. pneumoniae ssp. pneumoniae (2 isolates $-8.33 \%$ ), S. marcescens (17 isolates $-70.83 \%$ ) and $K$. oxytoca ( 5 isolates $-20.83 \%$ ). Characterization was made by biochemical tests of the automatized system, described in Table 3.

\subsection{Antibiotic susceptibility profiles}

The antibiotic susceptibility profiles of all 24 isolated bacteria were determined by VITEK ${ }^{\circledR} 2$ automated system and by anti-biogram AST cards, specific for Gram-negative bacteria.

Results showed that all isolates (100\%) were resistant to at least one of the 18 antibiotics tested by VITEK ${ }^{\circledR} 2$ automated system. All the identified bacteria of the same species showed the same susceptibility profile.

\section{Discussion}

\subsection{Biochemical conventional identification and biochemical identification by VITEK ${ }^{\circledR}$ Automated System}

Specific literature has shown that a great number of bacteria found in the effluent treatment systems are Gram-negative (Kanwar and Goswami, 2002). The most common are genus Acinetobacter spp. (Lima Junior, 2009), Aeromonas spp. (Ogino et al., 2000), Pseudomonas spp. (Sugimori et al., 2002), Burkholdeira spp. and Serratia spp. (Mohanan et al., 2007).

VITEK $^{\circledR} 2$ Automated System showed concordance within 19 isolates $(79.17 \%)$ and disagreement for five isolates $(20.83 \%)$ (Table 4$)$, when compared to results obtained in conventional biochemical tests. Current concordance results were different from those obtained by Bamford et al. (2010) when similar methodologies were compared. Clinical isolates environments.

All diagnoses when the two methodologies were used are described in table 4 . The first isolate that presented a difference in the identification was Bacterium 1 that in the conventional biochemical tests identified as $K$. oxytoca, whilst the VITEK ${ }^{\circledR} 2$ Automated System identified it as $K$. pneumoniae ssp. pneumoniae. For the differentiation of the two species, the degradation of ornithine, dulcitol and arabinose was made by conventional biochemical tests and, in turn, ornithine and 5-ketogluconate degradation was analyzed by VITEK ${ }^{\circledR} 2$ Automated System. These characteristics were studied by Drancourt et al. (2001) to differentiate species from Klebsiella and Raoutella genera.

$K$. oxytoca (isolate 13), identified belonging to the Enterobacteriaceae family were used in the above-mentioned study. The authors determined a higher concordance (93\%) in the results obtained when compared with those in our 
Table 1. Substrates present in the colorimetric identification card specific for Gram bacteria Fermenting or non fermenting (NG) negatives used in the VITEK 2 system.

\begin{tabular}{|c|c|c|c|}
\hline Well & Teste & Mnemonic & Amount/well (mg) \\
\hline 2 & Ala-Phe-Pro-ARYLAMIDASE & APPA & 0.384 \\
\hline 3 & ADONITOL & $\mathrm{ADO}$ & 0.1875 \\
\hline 4 & L-Pyrrolydonyl-ARILAMIDASE & PyrA & 0.018 \\
\hline 5 & L-Arabitol & IARL & 0.3 \\
\hline 7 & D-CELLOBIOSE & dCEL & 0.3 \\
\hline 9 & BETA-GALACTOSIDASE & BGAL & 0.036 \\
\hline 10 & H2S PRODUCTION & $\mathrm{H} 2 \mathrm{~S}$ & 0.0024 \\
\hline 11 & BETA-N-ACETYL-GLUCOSAMINIDASE & BNAG & 0.0408 \\
\hline 12 & GlutamylArylamidase pNA & AGLTp & 0.0324 \\
\hline 13 & D-Glucose & dGLU & 0.3 \\
\hline 14 & GAMMA-GLUTAMYL-TRANSFERASE & GGT & 0.0228 \\
\hline 15 & FERMENTATION?GLUCOSE & OFF & 0.45 \\
\hline 17 & BETA-GLUCOSIDASE & BGLU & 0.036 \\
\hline 18 & D-MALTOSE & Dmal & 0.3 \\
\hline 19 & D-MANNITOL & Dman & 0.1875 \\
\hline 20 & D-MANNOSE & Dmne & 0.3 \\
\hline 21 & BETA-XYLOSIDASE & Bxyl & 0.0324 \\
\hline 22 & BETA-ALANINEARYLAMIDASE pNA & BaLAP & 0.0174 \\
\hline 23 & L-Proline ARYLAMIDASE & ProA & 0.0234 \\
\hline 26 & LIPASE & LIP & 0.0192 \\
\hline 27 & PALATINOSE & PLE & 0.3 \\
\hline 29 & Tyrosine ARYLAMIDASE & TyrA & 0.0276 \\
\hline 31 & UREASE & URE & 0.15 \\
\hline 32 & D-SORBITOL & dSOR & 0.1875 \\
\hline 33 & SACCHAROSE/SUCRALOSE & SAC & 0.3 \\
\hline 34 & d-TAGATOSE & Dtag & 0.3 \\
\hline 35 & D-TREHALOSE & Dtre & 0.3 \\
\hline 36 & CITRATE (SODIUM) & CIT & 0.054 \\
\hline 37 & MALONATE & MNT & 0.15 \\
\hline 39 & 5-KETO-D-GLUCONATE & $5 \mathrm{KG}$ & 0.3 \\
\hline 40 & L-LACTATE alkalinisation & ILATk & 0.15 \\
\hline 41 & ALPHA-GLUCOSIDASE & AGLU & 0.036 \\
\hline 42 & SUCCINATE alkalinisation & SUCT & 0.15 \\
\hline 43 & Beta-N-ACETYL-GALACTOSAMINIDASE & NAGA & 0.0306 \\
\hline 44 & ALPHA-galactosidase & AGAL & 0.036 \\
\hline 45 & PHOSPHATASE & PHOS & 0.0504 \\
\hline 46 & Glycine Arylamidase & GlyA & 0.012 \\
\hline 47 & ORNITHINE DECARBOXYLASE & ODC & 0.3 \\
\hline 48 & LYSINE DECARBOXYLASE & LDC & 0.15 \\
\hline 52 & DECARBOXYLASE bASE & ODEC & $\mathrm{N} / \mathrm{A}$ \\
\hline 53 & L-HISTIDINE assimilation & IHISa & 0.087 \\
\hline 56 & COUMARATE & CMT & 0.126 \\
\hline 57 & BETA-GLUCORONIDASE & BGUR & 0.0378 \\
\hline 58 & O/129 RESISTANCE & O129R & 0.0105 \\
\hline 59 & Glu-Gly-Arg-ARYLAMIDASE & GGAA & 0.0576 \\
\hline 61 & L-MALATE assimilation & IMLTa & 0.042 \\
\hline 62 & ELLMAN & ELLM & 0.03 \\
\hline 64 & L-LACTATE assimilation & ILATa & 0.186 \\
\hline
\end{tabular}


Table 2. Results of biochemical tests performed to characterize genus of 24 Gram-negative bacteria isolated from residential, commercial and industrial effluents of region of Pelotas - RS.

\begin{tabular}{|c|c|c|c|c|c|c|c|c|c|c|c|c|c|c|}
\hline \multirow[b]{2}{*}{ BACTÉRIAS } & \multicolumn{14}{|c|}{ PROVAS BIOQUÍMICAS } \\
\hline & $\mathbf{C G}$ & $\mathbf{O X}$ & Mc & CI & SIM & $\mathbf{A R}$ & $\mathbf{C E}$ & RA & $\mathbf{X I}$ & $\mathbf{V P}$ & LAC & OR & $\mathbf{D U}$ & $\begin{array}{c}\text { Caracterização } \\
\text { bacteriana }\end{array}$ \\
\hline 1 & Gram - & - & + & + & -+- & + & + & + & + & + & + & - & + & K. oxytoca \\
\hline 2 & Gram - & - & + & + & --+ & - & $\mathrm{d}$ & - & $\mathrm{d}$ & + & - & + & - & S. marcescens \\
\hline 3 & Gram - & - & + & + & -+- & + & + & + & + & + & + & - & + & K. oxytoca \\
\hline 4 & Gram - & - & + & + & --+ & - & d & d & - & + & - & + & - & S. marcescens \\
\hline 5 & Gram - & - & + & + & --+ & - & - & - & - & + & - & + & - & S. marcescens \\
\hline 6 & Gram - & - & + & + & -+- & + & + & + & + & + & + & + & - & Raoutella sp. \\
\hline 7 & Gram - & - & + & + & --+ & - & - & - & - & + & - & + & - & S. marcescens \\
\hline 8 & Gram - & - & + & + & --+ & - & - & - & - & + & - & + & - & S. marcescens \\
\hline 9 & Gram - & - & + & + & --+ & - & + & - & - & + & - & + & - & S. marcescens \\
\hline 10 & Gram - & - & + & + & --+ & - & d & - & d & + & - & + & - & S. marcescens \\
\hline 11 & Gram - & - & + & + & --+ & - & d & - & d & + & - & + & - & S. marcescens \\
\hline 12 & Gram - & - & + & + & --+ & - & - & - & d & + & - & + & - & S. marcescens \\
\hline 13 & Gram - & - & + & + & -+- & + & + & + & + & + & + & - & + & K. oxytoca \\
\hline 14 & Gram - & - & + & + & --+ & - & - & - & d & + & - & + & - & S. marcescens \\
\hline 15 & Gram - & - & + & + & --+ & - & - & - & - & + & - & + & - & S. marcescens \\
\hline 16 & Gram - & - & + & + & --+ & - & - & d & d & + & $\mathrm{d}$ & + & - & S. marcescens \\
\hline 17 & Gram - & - & + & + & --+ & - & - & - & - & + & - & + & - & S. marcescens \\
\hline 18 & Gram - & - & + & + & --+ & - & - & - & - & + & - & + & d & S. marcescens \\
\hline 19 & Gram - & - & + & + & --+ & + & + & - & - & + & - & + & - & E. cloacae \\
\hline 20 & Gram - & - & + & + & -+- & + & + & + & + & + & + & + & + & Raoutella sp. \\
\hline 21 & Gram - & - & + & + & --+ & - & d & - & $\mathrm{d}$ & + & - & + & - & S. marcescens \\
\hline 22 & Gram - & - & + & + & --+ & - & - & - & - & + & - & + & - & S. marcescens \\
\hline 23 & Gram - & - & + & + & --- & - & + & + & + & + & + & + & - & K. pneumoniae \\
\hline 24 & Gram - & - & + & + & -+- & + & + & + & + & + & + & - & - & R. planticola \\
\hline
\end{tabular}

study, perhaps due to differences between isolated bacteria retrieved from different by VITEK $^{\circledR} 2$, showed negative ornithine, positive dulcitol and positive arabinose results, in agreement with results from the conventional tests for isolate 13 (K. oxytoca). On the other hand, biochemical test results of $K$. pneumoniae ssp. pneumoniae demonstrated positive ornithine, negative dulcitol and negative arabinose, disagreeing with results obtained in the conventional biochemical tests, but agreeing with the results obtained by VITEK $^{\circledR} 2$.

The two species from Raoutella genera, Raoutella planticola and Raoutella sp, identified by biochemical tests, showed discrepancy when compared with result obtained from VITEK $^{\circledR} 2$ Automated System, which identified all isolates as bacteria of species $K$. oxytoca.

Based on the 16s rRNA rpoB gene molecular sequence analysis, Drancourt et al. (2001) proposed that Klebsiella had to be divided into two genera, Klebsiella and Raoultella, and that $K$. oxytoca should be left as a monophyletic species. Beyond molecular analysis, the authors determined several differences between species from these genera, based on the biochemical test.

K. oxytoca bacterium is characterized by dulcitol, L-Tartrate and 3-O-Methyl-D-glucose negative degradation. However, bacteria Raoutella ornithinolytica, Raoutella planticola and Raoutella sp. are dulcitol and L-Tartrate negative and 3-O-Metil- $D$-glucose positive. In other words, they are typical characteristics that separate $K$. oxytoca from Raoutellas sp. (Drancourt et al., 2001).

Current research reveals that, through the use of conventional biochemical tests, Klebsiella and Raoutella should be separated, due to dulcitol degradation. VITEK ${ }^{\circledR} 2$ failed to perform the test. Conventional biochemical tests identified them as belonging to genus Raoutella. Results explain the discrepancy between the two methodologies used.

In the case of the 24 isolates, the two identification methodologies tests provided different results. The conventional biochemical tests identified the isolates as Enterobacter cloacae and the VITEK ${ }^{\circledR} 2$ automated system identified them as Serratia marcescens. In the biochemical tests, Enterobacter cloacae and Serratia marcescens were differentiated by arabinose and cellobiose degradation, although VITEK ${ }^{\circledR} 2$ only evaluated the cellobiose degradation.

Results demonstrated differences between the performed tests by $\mathrm{VITEK}^{\circledR} 2$ and conventional tests with regard to cellobiose degradation. Bacterium Enterobacter cloacae was cellobiose positive by conventional biochemical tests, whilst bacterium Serratia marcescens was cellobiose 
Table 3. Biochemical tests carried out to characterize 24 Gram negative bacteria isolated from effluents from food industries in the region of Pelotas - RS through VITEK ${ }^{\circledR} 2$ Automated System.

\begin{tabular}{|c|c|c|c|c|c|c|c|c|c|c|c|c|c|c|c|c|c|c|c|c|c|c|c|c|c|}
\hline & Test & 1 & 2 & 3 & 4 & 5 & 6 & 7 & 8 & 9 & 10 & 11 & 12 & 13 & 14 & 15 & 16 & 17 & 18 & 19 & 20 & 21 & 22 & 23 & 24 \\
\hline 1 & $\begin{array}{l}\text { Ala-Phe-Pro- } \\
\text { ARYLAMIDASE } \\
\text { (APPA) }\end{array}$ & - & - & - & - & - & - & - & - & - & - & - & - & - & - & - & - & - & - & - & - & - & - & - & - \\
\hline 2 & ADONITOL (ADO) & + & + & + & + & + & + & + & + & + & + & + & + & + & + & + & + & + & + & + & + & + & + & + & + \\
\hline 3 & $\begin{array}{l}\text { L-Pyrrolydonyl- } \\
\text { ARYLAMIDASE (Pyra) }\end{array}$ & + & + & + & + & + & + & + & + & + & + & + & + & + & + & + & + & + & + & + & + & + & + & + & + \\
\hline 4 & L-ARABITOL (IARL) & - & + & + & + & + & & + & + & + & + & + & + & + & + & + & + & - & + & + & + & + & - & + & + \\
\hline 5 & D-CELLOBIOSE (Dcel) & + & - & + & - & - & + & - & - & - & - & - & - & + & - & - & - & - & - & - & + & - & - & + & + \\
\hline 6 & $\begin{array}{l}\text { BETA- } \\
\text { GALACTOSIDASE } \\
\text { (BGAL) }\end{array}$ & + & - & + & - & - & + & - & - & - & - & - & - & + & - & - & + & - & - & - & + & - & - & + & + \\
\hline 7 & $\begin{array}{l}\mathrm{H}_{2} \mathrm{~S} \text { PRODUCTION } \\
\left(\mathrm{H}_{2} \mathrm{~S}\right)\end{array}$ & - & - & - & - & - & - & - & - & - & - & - & - & - & - & - & - & - & - & - & - & - & - & - & - \\
\hline 8 & $\begin{array}{l}\text { BETA-N-ACETYL- } \\
\text { GLUCOSAMINIDASE } \\
\text { (BNAG) }\end{array}$ & - & + & - & + & + & - & + & + & + & + & + & + & - & + & + & + & + & + & + & - & + & + & + & - \\
\hline 9 & $\begin{array}{l}\text { Glutamyl Arylamidase } \\
\text { pNA (AGLTp) }\end{array}$ & - & - & - & - & - & - & - & - & - & - & - & - & - & - & - & - & - & - & - & - & - & - & - & - \\
\hline 10 & D-GLUCOSE (Dglu) & + & + & + & + & + & + & + & + & + & + & + & + & + & + & + & + & + & + & + & + & + & + & + & + \\
\hline 11 & $\begin{array}{l}\text { GAMMA- } \\
\text { GLUTAMYL- } \\
\text { TRASFERASE (GGT) }\end{array}$ & - & - & - & - & - & - & - & - & - & - & - & - & - & - & - & - & - & - & - & - & - & - & + & - \\
\hline 12 & $\begin{array}{l}\text { FERMNTATION/ } \\
\text { GLUCOSE (OFF) }\end{array}$ & + & + & + & + & + & + & + & + & + & + & + & + & + & + & + & + & + & + & + & + & + & + & + & + \\
\hline 13 & $\begin{array}{l}\text { BETA-GLUCOSIDASE } \\
(\mathrm{BGLU})\end{array}$ & + & + & + & + & + & + & + & + & + & + & + & + & + & + & + & + & + & + & + & + & + & + & + & + \\
\hline 14 & D-MALTOSE (dMAL) & + & + & + & + & + & + & - & - & - & - & + & + & + & - & + & + & + & + & + & + & + & + & + & + \\
\hline 15 & $\begin{array}{l}\text { D-MANNITOL } \\
(\mathrm{dMAN})\end{array}$ & + & + & + & + & + & + & + & + & + & + & + & + & + & + & + & + & + & + & + & + & + & + & + & + \\
\hline 16 & D-MANNOSE (dMNE) & + & + & + & + & + & + & + & + & + & + & + & + & + & + & + & + & + & + & + & + & + & + & + & + \\
\hline 17 & $\begin{array}{l}\text { BETA-XYLOSIDASE } \\
\text { (BXYL) }\end{array}$ & - & - & + & - & - & + & - & - & - & - & - & - & + & - & - & - & - & - & - & + & - & - & + & + \\
\hline 18 & $\begin{array}{l}\text { BETA-Alanine } \\
\text { arylamidase pNA } \\
\text { (BAlap) }\end{array}$ & - & - & - & - & - & - & - & - & - & - & - & - & - & - & - & - & - & - & - & - & - & - & - & - \\
\hline 19 & $\begin{array}{l}\text { L-Proline } \\
\text { ARYLAMIDASE } \\
\text { (ProA) }\end{array}$ & - & + & - & + & + & - & + & + & + & + & + & + & - & + & + & + & + & - & + & - & + & - & - & - \\
\hline 20 & LIPASE (LIP) & - & - & - & - & - & - & - & - & - & - & - & - & - & - & - & - & - & - & - & - & - & - & - & - \\
\hline 21 & PALATINOSE (PLE) & + & - & + & - & - & + & - & - & - & - & - & - & + & - & - & - & - & - & - & + & - & - & + & + \\
\hline 22 & $\begin{array}{l}\text { Tyrosine } \\
\text { ARYLAMIDASE } \\
\text { (TyrA) }\end{array}$ & - & - & - & - & - & - & - & - & + & - & - & - & - & - & - & - & - & - & - & - & - & - & + & - \\
\hline 23 & UREASE (URE) & - & - & + & - & - & + & - & - & - & - & - & - & + & - & - & - & - & - & - & + & - & - & - & + \\
\hline 24 & D-SORBITOL (dSOR) & + & + & + & + & + & + & + & + & + & + & + & + & + & + & + & + & + & + & + & + & + & + & + & + \\
\hline 25 & $\begin{array}{l}\text { SACCHAROSE/ } \\
\text { SUCROSE (SAC) }\end{array}$ & + & + & + & + & + & + & + & + & + & + & + & + & + & + & + & + & + & + & + & + & + & + & + & + \\
\hline 26 & D-TAGATOSE (dTAG) & + & - & + & - & - & + & - & - & - & - & - & - & + & - & - & - & - & - & - & + & - & - & + & - \\
\hline 27 & $\begin{array}{l}\text { D-TREHALOSE } \\
\text { (dTRE) }\end{array}$ & + & + & + & + & + & + & + & + & + & + & + & + & + & + & + & + & + & + & + & + & + & + & + & + \\
\hline 28 & $\begin{array}{l}\text { CITRATE (SODIUM) } \\
\text { (CIT) }\end{array}$ & + & + & - & + & + & + & + & & + & + & + & + & + & + & + & + & + & + & + & + & + & + & + & + \\
\hline
\end{tabular}


Table 3. Continued...

\begin{tabular}{|c|c|c|c|c|c|c|c|c|c|c|c|c|c|c|c|c|c|c|c|c|c|c|c|c|c|}
\hline & Test & 1 & 2 & 3 & 4 & 5 & 6 & 7 & 8 & 9 & 10 & 11 & 12 & 13 & 14 & 15 & 16 & 17 & 18 & 19 & 20 & 21 & 22 & 23 & 24 \\
\hline 29 & MALONATE (MNT) & + & - & + & - & - & + & - & - & - & - & - & - & + & - & - & - & - & - & - & + & - & - & + & + \\
\hline ) & $\begin{array}{l}\text { 5-KETO-D- } \\
\text { GLUCONATE (5KG) }\end{array}$ & - & + & + & $(+)$ & + & + & - & - & - & $(-)$ & - & - & + & $(-)$ & + & + & $(-)$ & $(+)$ & + & + & + & $(-)$ & - & + \\
\hline 31 & $\begin{array}{l}\text { L-LACTATE } \\
\text { alkalinisation (ILATk) }\end{array}$ & + & + & - & - & + & + & - & - & + & - & - & + & + & + & - & - & + & + & + & + & - & - & + & + \\
\hline 32 & $\begin{array}{l}\text { ALPHA- } \\
\text { GALACTOSIDASE } \\
\text { (AGLU) }\end{array}$ & - & - & - & - & - & - & - & - & - & - & - & - & - & - & - & + & - & - & - & - & - & - & - & - \\
\hline 33 & $\begin{array}{l}\text { SUCCINATE } \\
\text { alkalinisation (SUCT) }\end{array}$ & - & + & - & - & + & + & - & - & - & - & + & + & + & + & + & + & + & - & + & + & + & + & + & + \\
\hline 34 & $\begin{array}{l}\text { Beta-N-ACETYL- } \\
\text { GALACTOSAMINIDASE } \\
\text { (NAGA) }\end{array}$ & - & + & - & + & + & - & + & + & + & + & + & + & - & + & + & + & + & + & + & - & + & + & - & - \\
\hline 35 & $\begin{array}{l}\text { ALPHA- } \\
\text { GALACTOSIDASE } \\
\text { (AGAL) }\end{array}$ & + & - & + & - & - & + & - & - & - & - & - & - & + & - & - & - & - & - & - & + & - & - & + & + \\
\hline 36 & $\begin{array}{l}\text { PHOSPHATASE } \\
\text { (PHOS) }\end{array}$ & - & - & + & - & - & + & - & - & + & - & - & - & + & - & - & - & - & - & + & + & - & - & + & + \\
\hline 37 & $\begin{array}{l}\text { Glycine } \\
\text { ARYLAMIDASE } \\
\text { (GlyA) }\end{array}$ & - & - & - & - & - & - & - & - & - & - & - & - & - & - & - & - & - & - & - & - & - & - & + & - \\
\hline 38 & $\begin{array}{l}\text { ORNITHINE } \\
\text { DECARBOXYLASE } \\
(\text { ODC) }\end{array}$ & - & + & - & + & + & - & + & + & + & + & + & + & - & + & + & + & + & + & + & - & + & + & - & - \\
\hline 39 & $\begin{array}{l}\text { LYSINE } \\
\text { DECARBOSYLASE } \\
\text { (LDC) }\end{array}$ & + & + & + & + & + & + & + & + & + & + & + & + & + & + & + & + & + & + & + & + & + & + & + & + \\
\hline 40 & $\begin{array}{l}\text { IDINE } \\
\text { tion (IHISa) }\end{array}$ & - & - & - & - & - & - & - & - & - & - & - & - & - & - & - & - & - & - & - & - & - & - & - & - \\
\hline 41 & ARATE (CMT) & + & + & - & + & + & + & + & + & + & + & + & + & - & + & + & + & + & + & + & + & + & + & + & - \\
\hline 42 & $\begin{array}{l}\text { BETA- } \\
\text { GLUCORONIDASE } \\
\text { (BGUR) }\end{array}$ & - & - & - & - & - & + & - & - & - & - & - & - & - & - & - & - & - & - & - & - & - & - & - & - \\
\hline 43 & $\begin{array}{l}\text { O/129 RESISTANCE } \\
\text { (comp.vibrio) (0129R) }\end{array}$ & + & + & + & + & - & + & $T$ & + & + & + & + & + & + & + & + & - & - & + & + & + & + & - & + & + \\
\hline 44 & $\begin{array}{l}\text { Glu-Gly-Arg- } \\
\text { ARYLAMIDASE } \\
\text { (GGAA) }\end{array}$ & - & + & - & + & - & - & + & + & + & + & + & + & - & + & + & + & + & + & + & - & + & + & - & - \\
\hline 45 & $\begin{array}{l}\text { L-MALATE } \\
\text { assimilation (IMLTa) }\end{array}$ & - & - & - & - & - & - & - & - & - & - & - & - & - & - & - & - & - & - & - & - & - & - & + & - \\
\hline 46 & ELLMAN (ELLM) & - & - & - & - & - & - & - & - & - & - & - & - & - & - & - & - & - & - & - & - & - & - & - & - \\
\hline 47 & $\begin{array}{l}\text { L-LACTATE } \\
\text { assimilation (ILATa) }\end{array}$ & - & - & - & - & - & - & - & - & - & - & - & - & - & - & - & - & - & - & - & - & - & - & - & - \\
\hline
\end{tabular}

negative. This fact corresponds to result by $\mathrm{VITEK}^{\circledR} 2$ and explains the difference in results obtained by the two methodologies.

The principal failures in VITEK ${ }^{\circledR} 2$ GNI card comprised carbohydrates, arabinose, cellobiose, sorbitol and arginine decarboxylation in all bacterial species (D'Azevedo et al., 2004).

A failure of a biochemical assimilation, due to a little inoculum or other factor can generate a different result that changes the final result. The conventional biochemical characterization performed in current study failed to reach up to the species identification of two of the $24(8.33 \%)$ identified isolates of the genus Raoutella sp. after researching the schemes proposed in the literature. The schemes used by different authors were based on isolates from clinical sources and little is known about the biochemical characterization of environmental isolates (Funke et al., 1997).

A crucial difficulty, associated with the bacterial species identification based on phenotypic characteristics, relies on 
Table 4. Comparison of the results of biochemical techniques of bacterial identification of bacteria isolated from effluents.

\begin{tabular}{|c|c|c|}
\hline N Isolated & Conventional test & VITEK $^{\circledR 2}$ \\
\hline 1 & K. oxytoca & K. pneumoniae ssp pneumoniae \\
\hline 2 & S. marcescens & S. marcescens \\
\hline 3 & K. oxytoca & K. oxytoca \\
\hline 4 & S. marcescens & S. marcescens \\
\hline 5 & S. marcescens & S. marcescens \\
\hline 6 & Raoutella sp. & K. oxytoca \\
\hline 7 & S. marcescens & S. marcescens \\
\hline 8 & S. marcescens & S. marcescens \\
\hline 9 & S. marcescens & S. marcescens \\
\hline 10 & S. marcescens & S. marcescens \\
\hline 11 & S. marcescens & S. marcescens \\
\hline 12 & S. marcescens & S. marcescens \\
\hline 13 & K. oxytoca & K. oxytoca \\
\hline 14 & S. marcescens & S. marcescens \\
\hline 15 & S. marcescens & S. marcescens \\
\hline 16 & S. marcescens & S. marcescens \\
\hline 17 & S. marcescens & S. marcescens \\
\hline 18 & S. marcescens & S. marcescens \\
\hline 19 & E. cloacae & S. marcescens \\
\hline 20 & Raoutella sp. & K. oxytoca \\
\hline 21 & S. marcescens & S. marcescens \\
\hline 22 & S. marcescens & S. marcescens \\
\hline 23 & K. pneumoniae & K. pneumoniae ssp pneumoniae \\
\hline 24 & R. planticola & K. oxytoca \\
\hline
\end{tabular}

the occurrence of divergence or convergence. Divergence occurs in some species lineages, genetically identical, but with different phenotypic characteristics. Convergence occurs in different species lineages, genetically different, but with a similar phenotypic behavior. In both situations, the phenotypic test resulted in a wrong identification (Siqueira Junior and Roças, 2005).

Although biochemical tests revealed high efficiency in environmental bacteria identification, as in Serratia marcescens, a convergence possibility in the tests cannot be discarded, according to the literature (Siqueira Junior and Roças, 2005), leading to discrepancies when conventional biochemical identification methods are compared.

The isolation and identification of new microorganisms with high enzyme production potential are greatly important since they exhibit a wide applicability and are efficient in the bioremediation process of contaminated places, beyond the efficient effluent treatment with high concentrations of oil and fats (Gopinath et al., 2013). A better development of identification techniques compatible with Environmental Microbiology laboratories is required.

\subsection{Antibiotic susceptibility profiles}

The antibiotic resistance in current analysis was high when compared to results by Zambrano et al. (2002). When these authors determined the antibiotic susceptibility profile from bacteria isolated from residential effluents, they reported that $90 \%$ of Enterobacteriaceae bacteria showed resistance to at least one antibiotic.

All isolates identified as S. marcescens $(100 \%)$ showed resistance to three kinds of antibiotics (cefuroxime, cefuroxime (axetil) and colistin), two of them belonging to the cephalosporin group (cefuroxime and cefuroxime (axetil)) in MIC $>=16$ and showed a sensitivity to the other antibiotics tested by VITEK ${ }^{\circledR} 2$ system.

Meanwhile, the bacterium identified as $K$. pneumonia spp. pneumonia showed resistance to two antibiotics (ampicillin in $\mathrm{MIC}=16$ and colistin in a $\mathrm{MIC}=4$ ) and was sensitive to the other antibiotics tested by VITEK ${ }^{\circledR} 2$ automated system. All isolates identified as $K$. oxytoca were resistant to one antibiotic (ampicillin) in $\mathrm{MIC}=16$ and were sensitive to all other antibiotics tested by $\mathrm{VITEK}^{\circledR} 2$ automated system.

Results contradicted those by Pereira (2013) who evaluated the antibiotic susceptibility profile for the following bacteria species: K. pneumonia, K. oxytoca and S. marcescens, isolated from Municipal Water Supply System of São José do Rio Preto SP Brazil.. The species failed to show any resistance to the antibiotics under analysis.

Current study showed a prevalent resistance to cephalosporin which belongs to the $\beta$-lactam class and interacts with penicillin-binding proteins (PBP), preventing the bacteria cell wall formation. Cephalosporin is one of the most common antibiotics used in different areas, such as human and veterinary medicine. Available for a long 
period on the market, the antibiotic ultimately has made selecting disseminating resistance factors or ending in selecting disseminating resistance factors. When antibiotics were discarded in the environment, they may be related to resistant microorganisms against these antibiotics (Oliveira, 2011).

In current study, it was not possible to observe a multi-resistant bacteria (Table 5) being a good microorganism characteristic being useful in the effluents treatment systems or in the bioremediation process. Several authors underscored that environmental multi-resistant bacteria populations, especially those in a contaminated environment, may increase due to the presence of resistant bacteria in industrial and hospital effluents and to the different types of genetic material exchange (Andersen, 1993), such as the horizontal gene transfer, providing an important risk to the human health.

Few studies are extant on the bacteria's susceptibility profile in effluents (Baquero et al., 2008). Li et al. (2009) isolated bacteria from effluents of a penicillin producer industry and evaluated their susceptibility profile. The authors underscored that some species of the Enterobacteriaceae family did showed penicillin resistance.

Li et al. (2010) determined the presence of oxytetracyclineresistant bacteria in effluents from industries producers of this antibiotic and also from a river in which the effluent was released. Analyses revealed the presence of species of the genus Klebsiella spp. and other oxytetracycline-resistant enterobacteria species.

Antibiotic-resistant bacteria reach the aquatic ecosystems through the activities of animals and people. These bacteria can capture resistant genes from carrier bacteria (Alonso et al., 2001). Further, many antibiotics from the industry are present in effluents that may reach aquatic ecosystems and disturb the original microbiota (Cabello, 2006).

Bacteria, including those in a natural environment without antibiotics, may transport a great number of resistance genes (Allen et al., 2009). It is difficult to explain the role of antibiotics in natural ecosystems from an anthropocentric point of view, since they are clinically based, or rather, an antibiotic efficiency to treat a particular infection or the removal of a resistance pathogenic agent.

To conclude about the importance of studying environmental bacteria and their susceptibility to antibiotics, this this work shows that to obtain efficient bacteria for the bioremediation process, some characteristics from isolated microorganisms should be taken into account. One characteristic is its identification to verify its pathogenicity and another the probability of dispersion

Table 5. Profile of susceptibility of bacteria of environmental origin identified by the VITEK2 System against antibiotics.

\begin{tabular}{cccccc}
\hline Isolado & Ampicilina & Cefuroxima & $\begin{array}{c}\text { Cefuroxoma } \\
\text { Axcetil }\end{array}$ & Colistina & $\begin{array}{c}\text { Piperacilina } \\
\text { tazobactam }\end{array}$ \\
\hline $\mathbf{1}$ & $\mathrm{R}$ & $\mathrm{S}$ & $\mathrm{S}$ & $\mathrm{R}$ & $\mathrm{S}$ \\
$\mathbf{2}$ & - & $\mathrm{R}$ & $\mathrm{R}$ & $\mathrm{R}$ & $\mathrm{S}$ \\
$\mathbf{3}$ & $\mathrm{R}$ & $\mathrm{S}$ & $\mathrm{S}$ & $\mathrm{S}$ & $\mathrm{S}$ \\
$\mathbf{4}$ & - & $\mathrm{R}$ & $\mathrm{R}$ & $\mathrm{R}$ & $\mathrm{S}$ \\
$\mathbf{5}$ & - & $\mathrm{R}$ & $\mathrm{R}$ & $\mathrm{R}$ & $\mathrm{S}$ \\
$\mathbf{6}$ & $\mathrm{R}$ & $\mathrm{S}$ & $\mathrm{S}$ & $\mathrm{S}$ & $\mathrm{S}$ \\
$\mathbf{7}$ & - & $\mathrm{R}$ & $\mathrm{R}$ & $\mathrm{R}$ & $\mathrm{S}$ \\
$\mathbf{8}$ & - & $\mathrm{R}$ & $\mathrm{R}$ & $\mathrm{R}$ & $\mathrm{S}$ \\
$\mathbf{9}$ & - & $\mathrm{R}$ & $\mathrm{R}$ & $\mathrm{R}$ & $\mathrm{S}$ \\
$\mathbf{1 0}$ & - & $\mathrm{R}$ & $\mathrm{R}$ & $\mathrm{S}$ \\
$\mathbf{1 1}$ & - & $\mathrm{R}$ & $\mathrm{R}$ & $\mathrm{R}$ & $\mathrm{S}$ \\
$\mathbf{1 2}$ & - & $\mathrm{R}$ & $\mathrm{R}$ & $\mathrm{S}$ \\
$\mathbf{1 3}$ & $\mathrm{R}$ & $\mathrm{S}$ & $\mathrm{R}$ & $\mathrm{S}$ & $\mathrm{S}$ \\
$\mathbf{1 4}$ & - & $\mathrm{R}$ & $\mathrm{R}$ & $\mathrm{S}$ \\
$\mathbf{1 5}$ & - & $\mathrm{R}$ & $\mathrm{R}$ & $\mathrm{R}$ & $\mathrm{S}$ \\
$\mathbf{1 6}$ & - & $\mathrm{R}$ & $\mathrm{R}$ & $\mathrm{R}$ & $\mathrm{S}$ \\
$\mathbf{1 7}$ & - & $\mathrm{R}$ & $\mathrm{R}$ & $\mathrm{S}$ \\
$\mathbf{1 8}$ & - & $\mathrm{R}$ & $\mathrm{R}$ & $\mathrm{R}$ & $\mathrm{S}$ \\
$\mathbf{1 9}$ & - & $\mathrm{R}$ & $\mathrm{R}$ & $\mathrm{S}$ \\
$\mathbf{2 0}$ & $\mathrm{R}$ & $\mathrm{S}$ & $\mathrm{R}$ & $\mathrm{S}$ & $\mathrm{S}$ \\
$\mathbf{2 1}$ & - & $\mathrm{R}$ & $\mathrm{R}$ & $\mathrm{R}$ & $\mathrm{S}$ \\
$\mathbf{2 2}$ & - & $\mathrm{R}$ & $\mathrm{S}$ & $\mathrm{R}$ & $\mathrm{S}$ \\
$\mathbf{2 3}$ & $\mathrm{R}$ & $\mathrm{S}$ & $\mathrm{S}$ & $\mathrm{S}$ & $\mathrm{S}$ \\
$\mathbf{2 4}$ & $\mathrm{R}$ & $\mathrm{S}$ & $\mathrm{S}$ & $\mathrm{S}$ \\
\hline
\end{tabular}

$\mathrm{S}=$ Sensitive; $\mathrm{R}=$ Resistant. Amicacina, Gentamicina, Cefepima, Cefoxitina, Ceftazidima, Ciprofloxacina, Tigeciclina, Ceftriaxona, Ampicilina Sulbactam, Meropenem, Ertapenem e Imipinem- All isolated were sensitive. 
of multi resistant bacteria. Coliform bacteria are a high diversity heterogeneous group in terms of genera and species, all belonging to the Enterobacteriaceae family (Edberg et al., 2000).

The microorganism's metabolism has the capacity to eliminate residues and to produce useful substances. Therefore, biodegradation consists in an important event to improve oil pollution in the environment, minimizing the environmental impact (Gopinath et al., 2013). Consequently, several microorganisms may be efficient in the treatment of contaminated places due to their biotechnological applications.

It can be concluded that the VITEK ${ }^{\circledR} 2$ automated system showed agreement on almost $80 \%$ of the samples identified and characterized by conventional identification systems. It is a system in question to identify environmental bacteria, but not satisfactory (over $90 \%$ ). The antibiotic susceptibility profile of bacteria isolated from effluents indicated resistance to at least one of the two antibiotics tested. And the absence of multiresistance and an important feature of isolated lipolytic bacteria that may possibly be used in effluent processes of residential, commercial and industrial bioremediation with high levels of lipids.

\section{References}

ABREU, T.E., PRETTO, A.J., OLIVEIRA, A., ARANHEN, R. and NAKAMURA, C., 2010. Avaliação da resistência a antibióticos de bactérias isoladas de efluente hospitalar. Acta Scientiarum. Technology, vol. 32, no. 1, pp. 1-5. http://dx.doi. org/10.4025/actascitechnol.v32i1.7453.

ALLEN, H.K., MOE, L.A., RODBUMRER, J., GAARDER, A. and HANDELSMAN, J., 2009. Functional metagenomics reveals diverse $\beta$-lactamases in a remote alaskan soil. The ISME Journal, vol. 3, no. 2, pp. 243-251. http://dx.doi.org/10.1038/ ismej.2008.86. PMid:18843302.

ALONSO, A., SÁNCHEZ, P. and MARTÍNEZ, J., 2001. Environmental selection of antibiotic resistance genes. Environmental Microbiology, vol. 3, no. 1, pp. 1-9. http://dx.doi.org/10.1046/j.14622920.2001.00161.x. PMid:11225718.

ANDERSEN, S., 1993. Effects of wastewater treatment on the species composition and antibiotic resistance of coliform bacteria. Current Microbiology, vol. 26, no. 2, pp. 97-103. http://dx.doi. org/10.1007/BF01577343.

BAMFORD, C., GOODWAY, J. and HOFFMANN, R., 2010. Rapid identification and susceptibility testing of Gram-negative bacilli from blood cultures using the Vitek ${ }^{\circledR} 2$ system. Southern African Journal of Infectious Diseases, vol. 25, no. 3, pp. 28-31.

BAQUERO, F., MARTINEZ, J.L. and CANTON, R., 2008. Antibiotics and antibiotic resistance in wáter environments. Current Opinion in Biotechnology, vol. 19, no. 3, pp. 260-265. http://dx.doi.org/10.1016/j.copbio.2008.05.006. PMid:18534838.

BARROW, G.I. and FELTHAM, R.K.A. Cowan and Steel's manual for the identification of medical bacteria. 3. ed. Cambridge: Cambridge University Press, 1993.216 p. http://dx.doi.org/10.1017/ CBO9780511527104.
BELLO, M.Y., 2007. Biological approach to oil spills remediation in the soil. African Journal of Biotechnology, vol. 6, no. 24, pp. 2735-2739. http://dx.doi.org/10.5897/AJB2007.000-2437.

BUSCH, U. and NITSCHKO, H., 1999. Methods for the differentiation of microorganisms. Journal of Chromatography. B, Biomedical Sciences and Applications, vol. 722, no. 1-2, pp. 263-278. http://dx.doi.org/10.1016/S0378-4347(98)00369-7. PMid:10068145.

CABELLO, F.C., 2006. Heavy use of prophylactic antibiotics in aquaculture: a growing problema for human and animal health and for the environment. Environmental Microbiology, vol. 8, no. 7, pp. 1137-1144. http://dx.doi.org/10.1111/j.1462-2920.2006.01054.x. PMid:16817922.

COLLA, L.M. and COSTA, J.A., 2003. Obtenção e aplicação de biossurfactantes. Vetor, vol. 13, pp. 85-103.

D'AZEVEDO, P.A., CANTARELLI, V., INAMINE, E., SUPERTI, S. and DIAS, G.A., 2004. Avaliação de um sistema automatizado na identificação de espécies de enterococcus. Jornal Brasileiro de Patologia e Medicina, vol. 40, no. 4, pp. 237-239.

DRANCOURT, M., BOLLET, C., CARTA, A. and ROUSSELIER, P., 2001. ROUSSELIER. Phylogenetic analyses of Klebsiella species delineate Klebsiella and Raoultella gen. nov., with description of Raoultella ornithinolytica comb. nov., Raoultella terrigena comb. nov. and Raoultella planticola comb. nov. International Journal of Systematic and Evolutionary Microbiology, vol. 51, no. 3, pp. 925-932. http://dx.doi.org/10.1099/00207713-51-3925. PMid:11411716.

EDBERG, S.C., RICE, E.W., KARLIN, R.J. and ALLEN, M.J., 2000. Escherichia coli: the best biological drinking water indicator for public health protection. Symposium Series (Society for Applied Microbiology), vol. 29, no. 29, pp. 106S-116. http:// dx.doi.org/10.1111/j.1365-2672.2000.tb05338.x. PMid:10880185.

FUNKE, G., VON GRAEVENITZ, A., CLARRIDGE 3RD, J.E. and BERNARD, K.A., 1997. Clinical microbiology of coryneform bacteria. Clinical Microbiology Reviews, vol. 10, no. 1, pp. 125-159. PMid:8993861.

GOPINATH, S.C.B., ANBU, P., LAKSHMIPRIYA, T. and HILDA, A., 2013. Strategies to characterize fungal lipases for applications in medicine and dairy industry. BioMed Research International, vol. 2013, pp. 1-10. http://dx.doi.org/10.1155/2013/154549. PMid:23865040.

GRAMINHA, E.B.N., GONÇALVES, A.Z.L., PIROTA, R.D.P.B., BALSALOBRE, M.A.A., SILVA, R. and GOMES, E., 2008. Enzyme production by solid-state fermentation: Application to animal nutrition. Animal Feed Science and Technology, vol. 144, no. 12, pp. 1-22. http://dx.doi.org/10.1016/j.anifeedsci.2007.09.029.

HASAN, F., SHAH, A.A. and HAMEED, A., 2006. Industrial applications of microbial lipases. Enzyme and Microbial Technology, vol. 39, no. 2, pp. 235-251. http://dx.doi.org/10.1016/j. enzmictec.2005.10.016.

KANWAR, L. and GOSWAMI, P., 2002. Isolation of a Pseudomonas lipase produced in pure hydrocarbon substrate and its application in the synthesis of isoamyl acetate using membraneimmobilised lipase. Enzyme and Microbial Technology, vol. 31, no. 6, pp. 727-735. http://dx.doi.org/10.1016/S0141-0229(02)00191-6.

LI, D., YANG, M., HU, J., ZHANG, J., LIU, R., GU, X., ZHANG, Y. and WANG, Z., 2009. Antibiotic resistance profile in environmental bacteria isolated from penicillin production wastewater treatment plant and the receiving river. Environmental 
Microbiology, vol. 11, no. 6, pp. 1506-1517. http://dx.doi. org/10.1111/j.1462-2920.2009.01878.x. PMid:19226301.

LI, D., YU, T., ZHANG, Y., YANG, M., LI, Z., LIU, M. and QI, R., 2010. Antibiotic resistance characteristics of environmental bacteria from an Oxytetracycline production wastewater treatment plant and the receiving river. Applied and Environmental Microbiology, vol. 76, no. 11, pp. 3444-3451. http://dx.doi. org/10.1128/AEM.02964-09. PMid:20400569.

LIMA JUNIOR, A.F. (2009). Biodegradação e atividade lipolitica em resíduos oleosos derivados do saneamento ambiental. Vitória: Universidade Federal do Espírito Santo. Dissertação.

MANDRI, T. and LIN, J., 2007. Isolation and characterization of engine oil degrading indigenous microrganisms in KwazuluNatal, South Africa. African Journal of Biotechnology, vol. 6, no. 1, pp. 23-27.

MARTINY, D., BUSSON, L., WYBO, I., EL HAJ, H., DEDISTE, A. and VANDENBERG, O., 2012. Comparison of the microflex lt and vitek ms systems for routine identification of bacteria by matrix-assisted laser desorption ionization-time of flight mass spectrometry. Journal of Clinical Microbiology, vol. 50, no. 4, pp. 1313-1325. http://dx.doi.org/10.1128/JCM.0597111. PMid:22322345.

MENDES, J.F., GONÇALVES, C.L., FERREIRA, G.F., ESTEVES, I.A., FREITAS, C.H., VILLARREAL, J.P.V., MELLO, J.R.B., MEIRELES, M.C.A. and NASCENTE, P.S., 2018. Antifungal susceptibility profile of diferent yeasts isolates from wild animals, cow?s milk with subclinical mastitis and hospital environment. Brazilian Journal of Biology $=$ Revista Brasileira de Biologia, vol. 8, no. 1, pp. 68-75. http://dx.doi. org/10.1590/1519-6984.04916.

MOHANAN, S., MARUTHAMUTHU, S., MUTHUKUMAR, N., RAJASEKAR, A. and PALANISWAMY, N., 2007. Biodegradation of palmarosa oil (green oil) by Serratia marcescens. International Journal of Environmental Science and Technology, vol. 4, no. 2, pp. 277-281. http://dx.doi.org/10.1007/BF03326285.

MONGKOLTHANARUK, W. and DHARMSTHITI, S., 2002. Biodegradation of lipidich wastewater by a mixed bacterial consortium. International Biodeterioration \& Biodegradation, vol. 50, no. 2, pp. 101-105. http://dx.doi.org/10.1016/S09648305(02)00057-4.

ODEYEMI, A.T., ADERIYE, B.I. and BAMIDELE, O.S., 2013. Lipolytic activity of some strains of Klebsiella, Pseudomonas and Staphylococcus spp. from restaurant wastewater and receiving stream. Journal of Microbiology Research (Rosemead, Calif.), vol. 3 , no. 1 , pp. 43-52.

OGINO, H., NAKAGAWA, S., SHINYA, K., MUTO, T., FUJIMURA, N., YASUDA, M. and ISHIKAWA, H., 2000. Purification and characterization of organic solvent stable lipase from organic solvent tolerant Pseudomonas aeruginosa LST-03. Journal of Bioscience and Bioengineering, vol. 89, no. 5, pp. 451-457. http://dx.doi.org/10.1016/S1389-1723(00)89095-7. PMid:16232776.

OLIVEIRA, V.D. 2011. Avaliação do perfil de resistência a antimicrobianos de bactérias gram-negativas isoladas nas águas do arroio Dilúvio. Rio Grande do Sul: Universidade Federal Do Rio Grande Do Sul, 84 p. Dissertação de Mestrado em Microbiologia Agrícola e do Ambiente.

OLIVO, V.A., LEOS, M.M. and CAMPOS, S.M., 2010. Extracción de grasas y aceites en los efluentes de una indústria automotriz. Consciência tecnológica, vol. 40, pp. 29-34.

PEIL, G.H., KUSS, A.V., RAVE, A.F., VILLARREAL, J.P., HERNANDES, Y.M. and NASCENTE, P.S., 2016. Bioprospecting of lipolytic microorganisms obtained from industrial effluents. Anais da Academia Brasileira de Ciências, vol. 88, no. 3, (suppl.), pp. 1769-1779. http://dx.doi.org/10.1590/0001-3765201620150550. PMid:27556331.

PEREIRA, O.L. 2013. Perfil de resistência aos antimicrobianos em coliformes isolados do Sistema Municipal de Abastecimento de Água de São José do Rio Preto - SP. São Paulo: Universidade Estadual Paulista "Júlio de Mesquita Filho", 83 p. Dissertação de Mestrado em Microbiologia.

POLONIO, C.J., POLLI, D.A., BULlA, C.M., ROSSETO, P., SANTOS, M.C., RHODEN, A.S., PAMPHILE, A.A. and CONTE, H. 2014. Potencial biorremediador de microrganismos: Levantamento de resíduos industriais e urbanos tratáveis no município de Maringá-PR. Biotechnology and Applied Biochemistry, vol. 3, no. 2, pp. 31-45.

REN, W.X., LI, P., GENG, Y. and LI, X., 2009. Biological leaching of heavy metals from a contaminated soil by Aspergillus niger. Journal of Hazardous Materials, vol. 167, no. 1-3, pp. 164-169. http://dx.doi.org/10.1016/j.jhazmat.2008.12.104. PMid:19232463.

ROMEU, B., SALAZAR, P., NAVARRO, A., LUGO, D., HERNÁNDEZ, U., ROJAS, N. and ESLAVA, C., 2010. Utilidad del sistema VITEK en la identificación y determinación de la susceptibilidad antimicrobiana de bacterias aisladas de ecosistemas dulceacuícolas. CENIC, vol. 41, pp. 1-9.

SIQUEIRA JUNIOR, J.F. and ROÇAS, I.N., 2005. Exploiting molecular methods to explore endodontic infections: part 1current molecular technologies for microbiological diagnosis. Journal of Endodontics, vol. 31, no. 6, pp. 411-423. http://dx.doi. org/10.1097/01.don.0000157989.44949.26. PMid:15917679.

SONG, B. and LEFF, L., 2005. Identification and characterization of bacterial isolates from the Mir space station. Microbiological Research, vol. 160, no. 2, pp. 111-117. http://dx.doi.org/10.1016/j. micres.2004.10.005. PMid:15881827.

SUGIMORI, D., NAKAMURA, M. and MIHARA, Y., 2002. Microbial degradation of lipid by Acinetobacter sp. strain SOD1. Journal of Bioscience, Biotechnology, and Biochemistry, vol. 66, no. 7, pp. 1579-1582. http://dx.doi.org/10.1271/bbb.66.1579. PMid:12224647.

ZAMBRANO, J.L., BOTERO, L., CAVAZZA, M.E. and AVILA, M., 2002. Resistencia a antimicrobianos y presencia de plásmidos en cepas de Escherichia coli aisladas de aguas residuales crudas y tratadas de lagunas de estabilización con fines de reuso en agricultura. Revista de la Sociedad Venezolana de Microbiología, vol. 22 , no. 1 , pp. 44-50. 